\title{
THE EVOLUTION OF AGB STARS
}

\author{
P.R. WOOD AND E. VASSILIADIS \\ Mount Stromlo and Siding Spring Observatories \\ Private Bag, Weston Creek P.O. \\ Canberra, ACT 2611 \\ Australia
}

\section{Introduction}

Computations of AGB stellar evolution which include the effects of mass loss are still relatively rare. However, in order to relate numbers of Mira variables, OH/IR stars and carbon stars to associated stellar populations, it is necessary to understand evolutionary timescales on the AGB.

The dominant factors controlling very late AGB evolution are shell flashes and mass loss, and some quantitative estimate of the latter is needed for stellar evolution calculations. The favoured mechanism for the production of the large mass loss rates observed in late AGB stars such as OH/IR stars and dust-enshrouded carbon stars, which have mass loss rates up to a few times $10^{-5} \mathrm{M}_{\odot} \mathrm{yr}^{-1}$ (see van der Veen and Rugers 1989 for a compilation), is a dual process involving the levitation of matter above the photosphere by large-amplitude radial pulsation followed by the formation of grains on which radiation pressure acts to drive the circumstellar material away from the star (Castor 1981; Holzer and MacGregor 1985; Hearn 1990). The studies by Wood (1979) and Bowen (1988) show that, by themselves, neither pulsation nor radiation pressure acting on grains can produce the very large mass loss rates from AGB stars.

\section{Timescales for AGB evolution}

Since it is thought that the mass loss rates in AGB stars are determined by pulsation of the central star, we have adopted the formula for mass loss rate as a function of pulsation period given by Wood (1990); this empirical formula is based on CO microwave observations of circumstellar winds.

A first approach to studying late AGB evolution is to ignore helium shell flashes and to use the well known, and much used, luminosity-core mass relation which implies a constant rate of AGB evolution of $\sim 1 \mathrm{mag}$. per $10^{6}$ years. Such an approach was taken by Wood (1990). The observed Mira lifetimes of $\sim 7 \times 10^{4}$ years (Wood 1990; Hughes and Wood 1990), or even the larger lifetime of $\sim 2 \times 10^{5}$ years derived for local Miras by Jura and Kleinmann (1991), is difficult to explain with such simple models (Wood 1990).

The major simplification in the calculations of Wood (1990) was the neglect of helium shell flashes. Figure 1 shows the evolution of a $1 \mathrm{M}_{\odot}$ AGB stars in the presence of both shell flashes and mass loss. It is immediately clear that over the last $\sim 5 \times 10^{5}$ years of AGB evolution, evolutionary rates are nothing like the mean rate of $\sim 1$ mag. per $10^{6}$ years: evolution rates are dominated by helium shell flash behaviour. In fact, a star at a random position on the AGB is more likely to be evolving at a rate of $\sim 1 \mathrm{mag}$. per $10^{5}$ years, corresponding to the mean rate of recovery of luminosity between flashes. With that kind of evolutionary rate it is entirely plausible that a typical low mass Mira variable (such as the 


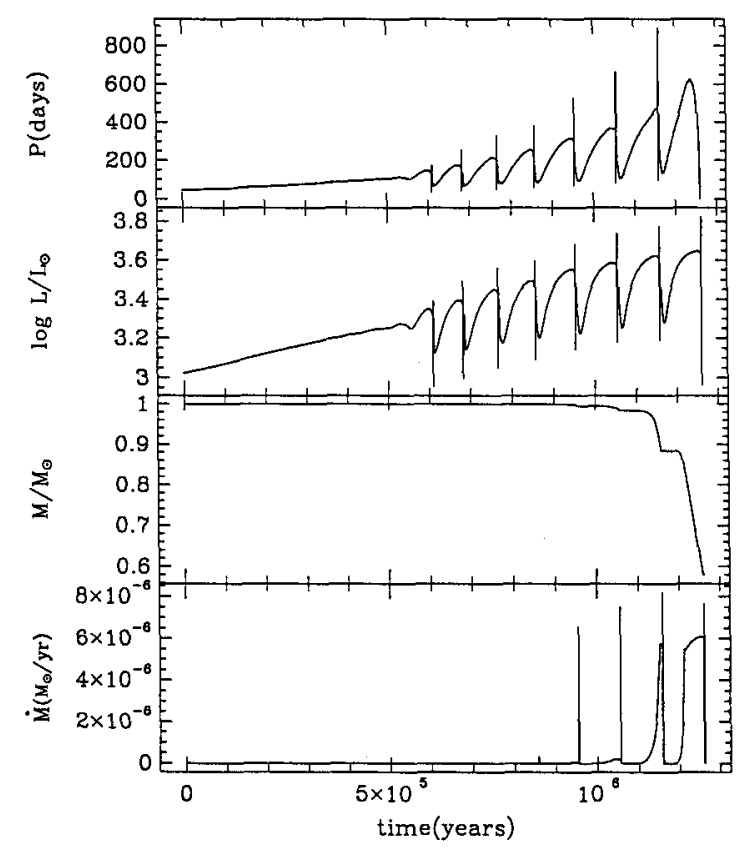

Figure 1. The pulsation period $\mathrm{P}$, luminosity $\mathrm{L}$, mass $\mathrm{M}$ and mass loss rate $\dot{\mathrm{M}}$ plotted against time on the AGB for a $1 \mathrm{M}_{\odot}$ star with LMC abundance.

prototype o Ceti with $\mathrm{P} \sim 330$ days) could increase its period sufficiently over $\sim 10^{5}$ years to turn into a dusty $\mathrm{OH} / \mathrm{IR}$ star and leave the AGB to become a planetary nebula.

The behaviour of the period, stellar mass and mass loss rate during the AGB phase is also shown in Figure 1. A notable feature of these calculations is the way in which the mass loss rate suddenly runs away during the last two shell flashes, causing the stellar mass to drop rapidly in two steps. The mass loss runaway is due partly to the increase in luminosity (and hence radius) and partly to the decrease in mass which directly affects the period through the pulsational period-mass-radius relation $P \sim R^{2} / M$ (Wood 1990). Behaviour such as that in Figure 1 means that, following a helium shell flash, many of the AGB stars that are currently surrounded by thick circumstellar shells may not directly turn into planetary nebulae but may settle back down on the AGB again with small mass loss rates and dissipated circumstellar shells.

\section{Wind models}

In conjunction with the above calculations, a hydrodynamic code was used to study the time dependence of the stellar winds and circumstellar envelopes formed as a result of the mass loss produced on the AGB. The results for a typical AGB star are shown in Figure 2. Two interesting results of the calculations are (1) a considerable number of AGB stars may have hollow circumstellar shells due to the sudden drop in mass loss rate at a helium shell flash, and in many of these shells matter may be falling inwards rather than flowing outwards, and (2) at the luminosity peak associated with helium shell flashes, a faster wind may be driven into the pre-existing 'superwind'. In the latter case, there should be evidence for winds at two velocities in the $1612 \mathrm{MHz}$ profiles of OH/IR stars, although such objects should be rare ( $\sim 1 \%$ of OH/IR stars). The objects 


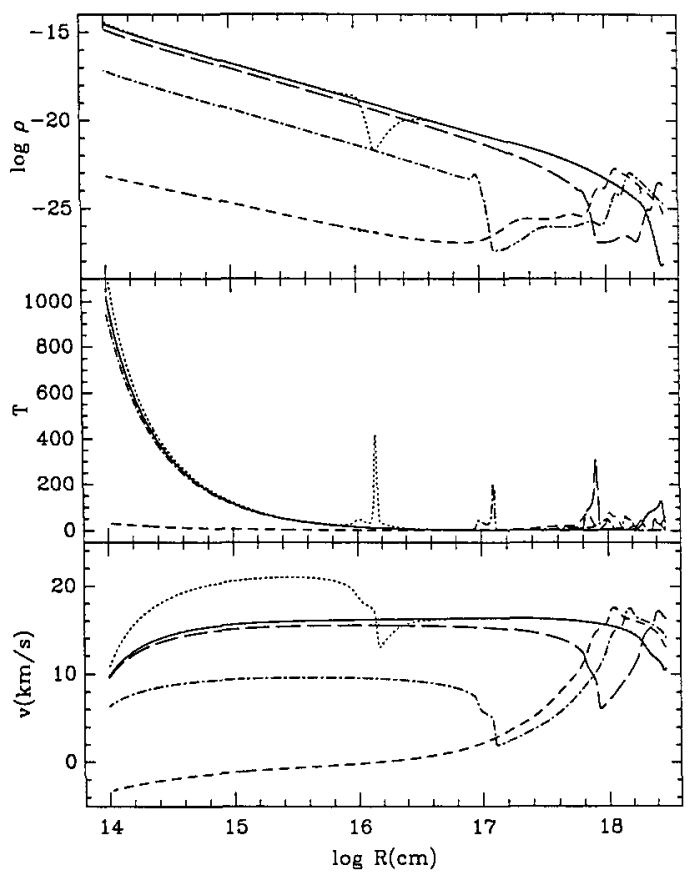

Figure 2. Density $\rho$, temperature $T$ and velocity $v$ of material in stellar winds around an AGB star as a function of distance $R$ from the star. The structure is shown just before a helium shell flash (continuous line), at the luminosity peak of a shell flash (dotted line), at luminosity minimum of the flash cycle (short dashes) and during the rise to the next quiescent luminosity maximum (dot-dash and long dash lines).

$18520+0533$ in Eder, Lewis and Terzian (1988) and 17253-2824 in te Lintel Hekkert (1990) show just such structure. A fuller account of the above results will appear elsewhere (Vassiliadis and Wood 1992, in preparation).

\section{References}

Bowen, G. 1988, Ap.J., 329, 844.

Castor, J.I. 1981, in Physical Processes in Red Giants, eds. I. Iben and A. Renzini (Reidel), p.285.

Eder, J., Lewis, B.M. and Terzian, Y. 1988, Ap.J. Suppl., 66, 183.

Hearn, A.G. 1990, in From Miras to Panetary Nebulae: Which Path Stellar Evolution?, eds. M.O. Mennessier and A. Omont (Editions Frontières), p.121.

Holzer, T.E. and MacGregor, K.B. 1985, in Mass Loss from Red Giants, eds. M. Morris and B. Zuckerman (Reidel), p.229.

Hughes, S.M.G and Wood, P.R. 1990, A.J., 99, 784.

Jura, M. and Kleinmann, S.G. 1991, preprint.

te Lintel Hekkert, P. 1990, thesis, Leiden University.

van der Veen, W.E.C.J. and Rugers, M. 1989, Astr. Ap., 226, 183.

Wood, P.R. 1979, Ap.J., 227, 220.

Wood, P.R. 1990, in From Miras to Panetary Nebulae: Which Path Stellar Evolution?, eds. M.O. Mennessier and A. Omont (Editions Frontières), p.67. 